\title{
Stress gradients in CrN coatings
}

\author{
G. C. A. M. Janssen ${ }^{\text {a) }}$ \\ Department of Materials Science and Engineering, Delft University of Technology, Mekelweg 2, 2628CD \\ Delft, The Netherlands \\ F. D. Tichelaar \\ Kavli Institute of Nanoscience, Delft University of Technology, Lorentzweg 1, 2628 CJ Delft, \\ The Netherlands \\ C. C. G. Visser \\ Dimes, Delft University of Technology, Mekelweg 4, 2628 CD Delft, The Netherlands
}

(Received 7 June 2006; accepted 2 August 2006; published online 8 November 2006)

\begin{abstract}
Stress in hard films is the net sum of tensile stress generated at the grain boundaries, compressive stress due to ion peening, and thermal stress due to the difference in thermal expansion of the coating and substrate. The tensile part due to grain boundaries is thickness dependent. The other two contributions are not thickness dependent. Summation of the three components leads to a stress gradient in the coating. In the present paper it is demonstrated that adding the three contributions mentioned above yields a good description of the observed dependence of stress on thickness in CrN coatings. (C) 2006 American Institute of Physics. [DOI: 10.1063/1.2363818]
\end{abstract}

\section{INTRODUCTION}

In order to discuss the occurrence of stress in thin films, it is useful to distinguish films made from materials that do reconstruct during growth (typically low melting point fcc materials) and films that do not reconstruct during growth, (typically high melting point metals and ceramics). The distinction is not absolute, the important quantity is the homologous deposition temperature $T_{\text {dep }} / T_{\text {melt }}{ }^{1,2}$

For films deposited at a high $T_{\text {dep }} / T_{\text {melt }}$ ratio the grain boundaries are perpendicular to the surface and the width of the grains increases with increasing film thickness. ${ }^{1,2}$ For aluminum deposited at room temperature $T_{\text {dep }} / T_{\text {melt }}=0.31$. At this homologous temperature an intermediate grain structure is obtained. ${ }^{3}$ This grain structure implies lateral grain growth during film growth and makes the description of stress development with thickness quite a challenge. The problem becomes even more complicated when one realizes that the observed stresses are of the same order as the yield stress of the film material. Extensive experiments have been performed on stress in these films. ${ }^{4-6}$ Even a model with microstructure dependent stress relaxation has been offered. ${ }^{5}$ This model captures the experimentally observed effects, but it lacks the simplicity of the description by Doljack and Hoffman. ${ }^{7}$

Stress development in films made out of high melting point materials is much more traceable. In those films the grain boundaries are formed at the arrival of the atoms at the growing surface of the film. The resulting grain structure has been captured in the zone model by Movchan and Demchishin as zone $T$. The zone model, especially zone $T$, has been elaborated on both experimentally ${ }^{2}$ as well as theoretically.,

Recently, we demonstrated the existence of a stress gradient in Cr thin films. ${ }^{10}$ In that paper it was shown that the

\footnotetext{
a) Author to whom correspondence should be addressed; electronic mail: g.c.a.m.janssen@tudelft.nl
}

tensile stress at height $z$ in the film was directly proportional to the number of grains per unit length at height $z$. Since the number of grains per unit length at height $z$ shows a power law dependence on the height $z$, so does the tensile stress at height $z$. The total stress in the film is the integral of the stress at height $z$ over the thickness divided by the thickness. In Ref. 10 it is shown that the total tensile stress shows a power law dependence on film thickness. The physics underlying this proportionality of tensile stress and number of grain boundaries per unit length is the grain boundary shrinking, as introduced by Doljack and Hoffman in their seminal paper. $^{7}$

Compressive stress is caused by ion peening. ${ }^{11,12}$ An ion bombardment on the growing film induces defects, either argon or self-interstitials, leading to compressive stress. In $\mathrm{x}$-ray diffraction on chromium films we observed an increase in lattice parameters in all directions with respect to the values calculated for a biaxial compressive stress. ${ }^{13}$ For sputter deposited chromium films we showed that the tensile stress originating at the grain boundaries and the tensile stress due to the ion induced defects are additive. ${ }^{13}$

$\mathrm{Me}-\mathrm{N}$ films are of extreme technological importance, e.g., TiN, TaN, TiAlN, and CrN. Unfortunately, for these thin films it is less easy to isolate the various contributions to the stress. In order to obtain good quality films, deposition has to be done at elevated temperature while applying a bias voltage to the substrate. In the present paper we show that the description developed for chromium can also be applied to CrN.

\section{EXPERIMENT}

Chromium nitride films were deposited in a Hauzer HC 750 deposition machine. The films were deposited on $100 \mathrm{~mm}$ diameter Si wafers with 100 orientation by reactive sputter deposition in an argon nitrogen plasma from a chromium target. The dimensions of the target were 120 


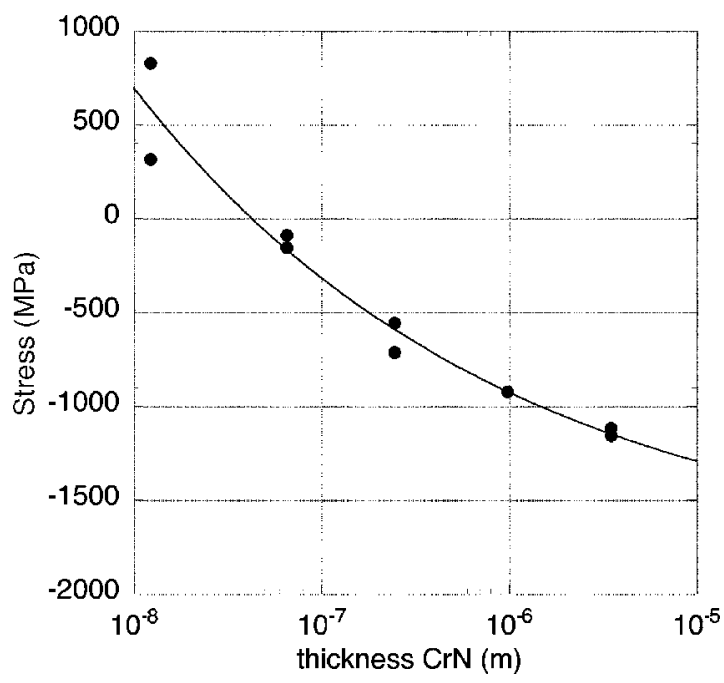

FIG. 1. Stress in $\mathrm{CrN}$ coatings deposited at $450{ }^{\circ} \mathrm{C}$ with a bias of $175 \mathrm{~V}$. The curve is a fit to the data by a power law plus a thickness independent term.

$\times 600 \mathrm{~mm}^{2}$ and the power applied to the target was $5 \mathrm{~kW}$. The bias applied to the samples was $175 \mathrm{~V}$. This value was chosen based on Ref. 12, in which for TiN it was shown that below $200 \mathrm{~V}$ bias growth in zone $T$ occurs. A higher bias voltage might lead to renucleation. During deposition a second plasma source was engaged in order to obtain a sufficiently high plasma density to ensure not only a bias voltage but also a sufficient bias current to warrant the deposition of dense films. ${ }^{14}$ The argon flow was 115 SCCM (SCCM denotes cubic centimeter per minute at STP) in all experiments. The nitrogen flow was either 50 or 70 SCCM. The pressure during deposition was about $0.4 \mathrm{~Pa}$. The samples performed a planetary motion in front of the target. The chamber was heated by resistive heaters to $450{ }^{\circ} \mathrm{C}$ during deposition. Two series of films $\left(\mathrm{N}_{2}\right.$ flow either 50 or $\left.70 \mathrm{SCCM}\right)$ ranging in thickness from $12 \mathrm{~nm}$ to $3.5 \mu \mathrm{m}$ were deposited using these parameters.

Stress was measured by wafer curvature. ${ }^{15}$ The curvature and weight of the wafer was measured before and after deposition. In both measurements the curvature was measured versus an optical flat in order to avoid errors due to misalignment of the lasers. Thermal stress was measured by reheating the wafer after deposition from RT to $400{ }^{\circ} \mathrm{C}$ while at the same time observing the curvature of the wafer. Composition was checked by electron probe micro analysis (EPMA); crystallinity and phases were checked by x-ray diffraction. Microstructure was observed by transmission electron microscopy (TEM). A flow of 50 SCCM of nitrogen led to films with composition of $\mathrm{Cr}_{54} \mathrm{~N}_{46}$. A nitrogen flow of 70 SCCM led to films with a composition of $\mathrm{Cr}_{51} \mathrm{~N}_{49}$. X-ray diffraction on films from both series showed only $\mathrm{CrN}$ peaks.

\section{RESULTS}

In Fig. 1 the average stress in the film as a function of film thickness $h$ in the $\mathrm{CrN}$ films deposited with a nitrogen flow of 70 SCCM is presented. The data have been fitted by

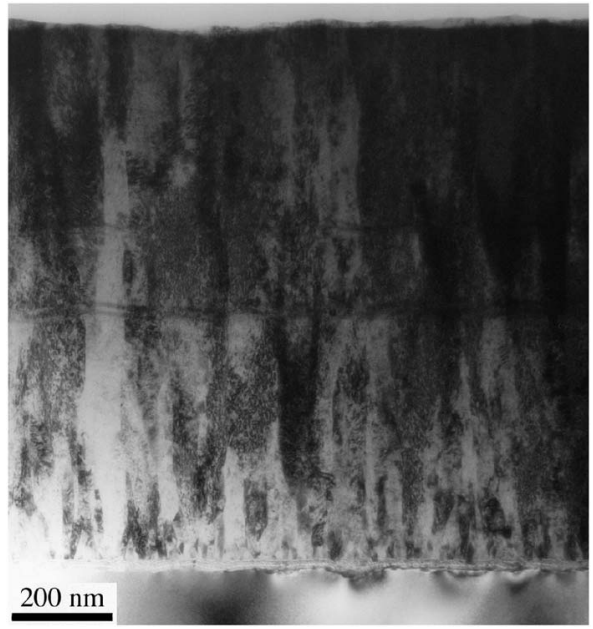

FIG. 2. TEM cross section of a $1 \mu \mathrm{m}$ thick $\mathrm{CrN}$ coating.

$\sigma_{\mathrm{av}}(h)=C_{1}+C_{2} h^{-p}$,

with $h$ the thickness of the film and $p$ the exponent that describes the evolutionary grain growth.

From the fit we obtained $C_{1}=-1.80 \mathrm{GPa}$, the limiting value for stress in thick films, $C_{2}=44 \mathrm{MPa} \mathrm{m}^{0.22}$, a number proportional to the amount of stress generated per grain boundary, and $p=0.22$.

In Fig. 2(a) TEM cross section of a $\mathrm{CrN}$ film deposited with a $\mathrm{N}_{2}$ flow of 70 SCCM is shown. The development of the microstructure, with many grains at the bottom of the film and less grains at the top, is clearly visible. Also visible are two horizontal bands at half height and 5/8th height. Those bands are caused by a failure of the second plasma source during deposition. Locally, the density of the film will be somewhat lower, but the evolution of the grain structure is seen to be unaffected. From this TEM micrograph the development of grain size $(w)$ with height in the film $z$ is analyzed (Fig. 3). A power law fit, with a power prescribed by the data in Fig. 2, is made,

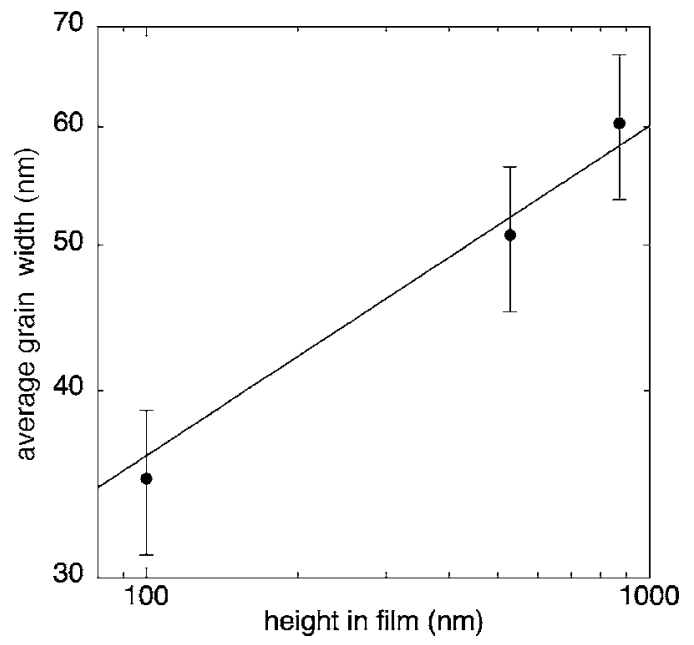

FIG. 3. Grain width vs height in the film. The curve is a power law fit to the data points with the exponent obtained from the fit to the stress data in Fig. 2. 


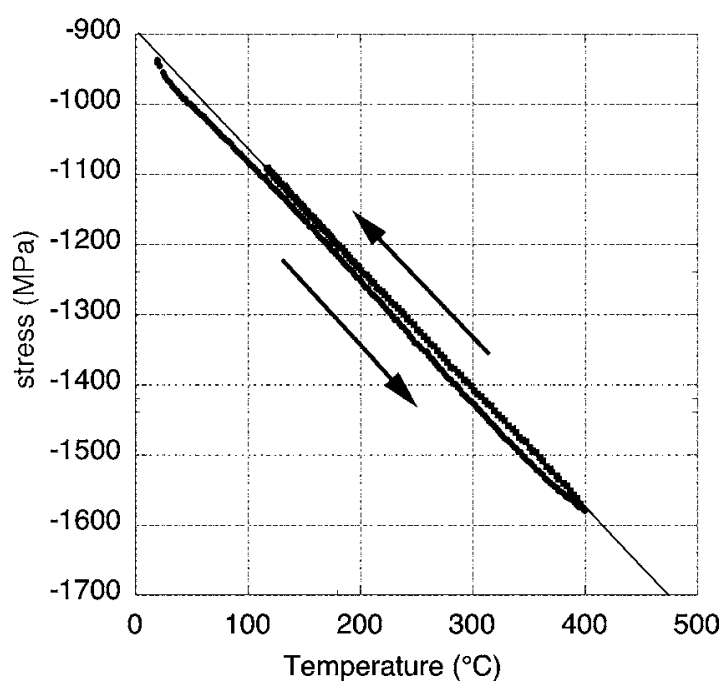

FIG. 4. Stress in a $1 \mu \mathrm{m}$ thick CrN coating on silicon during reheating and cooling.

$$
w(z)=C_{3} z^{p}
$$

with $C_{3}=1.25 \times 10^{-6} \mathrm{~m}^{0.78}$.

As stated before, the total stress is the sum of the contributions by grain boundaries, ion peening, and thermal expansion. Of these three the contribution by the grain boundaries is easily isolated since it is the only contribution that depends on thickness. The other two contributions are thickness independent and need a separate experiment to be isolated. After deposition and cooling to room temperature, we reheated a wafer with a $0.98 \mu \mathrm{m}$ thick $\mathrm{CrN}$ film to $400{ }^{\circ} \mathrm{C}$ while measuring the curvature and hence the stress. In Fig. 4 the data are presented. The data points during heating and cooling almost coincide, indicating little relaxation or oxidation of the film. From the data points obtained during cooling the coefficient of thermal expansion of $\mathrm{CrN}$ can be determined. $^{16}$

$$
\alpha_{\mathrm{CrN}}=\alpha_{\mathrm{Si}}-\frac{d \sigma / d T}{[E /(1-\nu)]_{\mathrm{CrN}}} .
$$

Taking $\nu_{\mathrm{CrN}}=0.25$, combined with $E /\left(1-\nu^{2}\right)=280 \mathrm{GPa}$ reported for these films earlier, ${ }^{14}$ leads to $[E /(1-\nu)]_{\mathrm{CrN}}$ $=373 \mathrm{GPa}$. With $\alpha_{\mathrm{Si}}=2.6 \times 10^{-6} \mathrm{~K}^{-1}$ (Ref. 17) and the measured value of $d \sigma / d T=-1.70 \mathrm{MPa} / \mathrm{K}$, this leads to $\alpha_{\mathrm{CrN}}$ $=7.16 \times 10^{-6} \mathrm{~K}^{-1}$.

The contribution of thermal expansion due to the elevated deposition temperature of $450{ }^{\circ} \mathrm{C}$ to the room temperature stress follows directly from the slope of $d \sigma / d T$ multiplied by $\Delta T$ and is $731 \mathrm{MPa}$ tensile. From the fit of Eq. (1) to the thickness dependent stress measurements, we obtained $-1.85 \mathrm{GPa}$ as a limiting value for the stress in thick films. Combining this number with the thermal stress leads to an ion peening contribution of $-2.58 \mathrm{GPa}$ to the total stress.

In Fig. 5 the stress thickness dependence of $\mathrm{CrN}$ films deposited at a nitrogen flow of 50 SCCM is presented. Apparently, stress in understoichiometric $\mathrm{CrN}$ films develops with thickness comparable to stress in stoichiometric films. The data can be fitted by the same power law that was used for the stoichiometric films. The limiting value of compres-

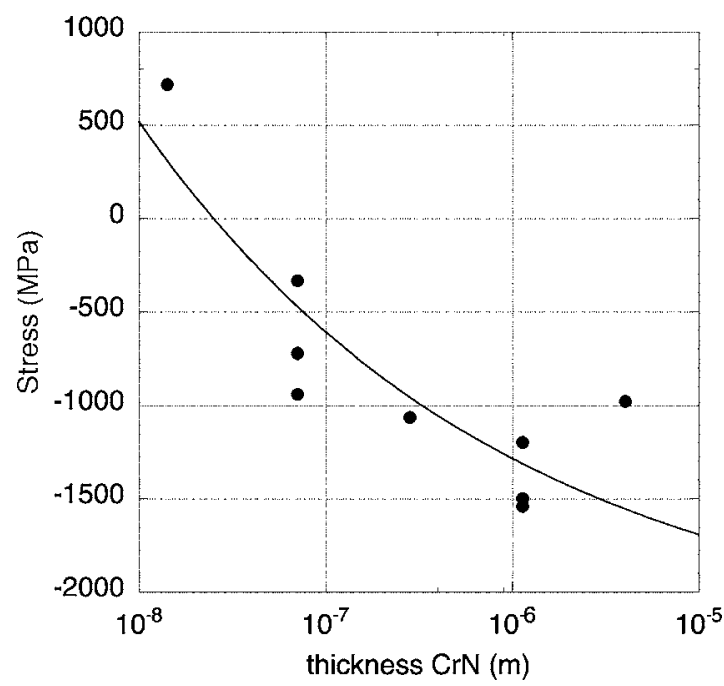

FIG. 5. Stress in an understoichiometric CrN coating. The fit is by power law plus thickness independent term. The exponent in the power law is taken from the fit in Fig. 3.

sive stress is larger than in the case of stoichiometric $\mathrm{CrN}$, $-2.69 \mathrm{vs}-1.85 \mathrm{GPa}$. The scatter in the data, however, is also larger; therefore no conclusion is drawn from this difference. A TEM micrograph of the understoichiometric $\mathrm{CrN}$ film showed less smooth grain boundaries, in line with the scatter in the stress data.

\section{DISCUSSION}

The thermal expansion coefficient of $\mathrm{CrN}$ has been determined from wafer curvature measurements at temperatures between RT and $400{ }^{\circ} \mathrm{C}$. At RT the coated side of the wafer was curved convex, indicating a compressive stress in the $\mathrm{CrN}$ film. Upon heating the radius of curvature became smaller, indicating $\alpha_{\mathrm{CrN}}>\alpha_{\mathrm{Si}}$. This was no small effect (see Fig. 4). Since $\alpha_{\mathrm{Si}}=2.6 \times 10^{-6} \mathrm{~K}^{-1}$, we believe the values reported previously for $\alpha_{\mathrm{CrN}} 0.7 \times 10^{-6}$ (Ref. 18) and 2.3 $\times 10^{-6}$ (Ref. 19) to be inaccurate. The high temperature value $\left(850-1040^{\circ} \mathrm{C}\right.$ ) for $\alpha_{\mathrm{CrN}}=7.5 \times 10^{-6}$ reported in (Ref. 19) is much closer to the value we report: $7.16 \times 10^{-6} \mathrm{~K}^{-1}$. We suppose that the reported low values for $\alpha_{\mathrm{CrN}}$ have been obtained on porous films. In (Ref. 18) a hardness of $10 \mathrm{GPa}$ is reported, characteristic of porous films. Even though the value for $E_{r}$ reported in (Ref. 14) coincides with other reports cited in that paper, $E_{\mathrm{CrN} \text {,poly }}=280 \mathrm{GPa}$ is much smaller than $E_{\mathrm{CrN}, 001}=405 \mathrm{GPa}$ reported in (Ref. 20). If that value were to be used in calculating the coefficient of thermal expansion from our measurements, one would arrive at $\alpha_{\mathrm{CrN}}$ $=5.75 \times 10^{-6} \mathrm{~K}^{1}$.

Combining Eqs. (1) and (2), we arrive at an expression for the amount of grain boundary shrinking $\Delta$ associated with the tensile stress. ${ }^{10}$

$$
\Delta=\frac{\left(1-\nu_{\mathrm{CrN}}\right)(1-p)}{E_{\mathrm{CrN}}} C_{2} C_{3} .
$$

Entering fit values and constants in Eq. (4) yields $\Delta_{\mathrm{CrN}}$ $=0.08 \mathrm{~nm}(27 \%$ of the $\mathrm{Cr}-\mathrm{Cr}$ distance in $\mathrm{CrN})$. This number is higher than the number obtained for chromium films, $0.013 \mathrm{~nm}$ ( $5 \%$ of the interatomic distance $)^{10}$ but equal to the 
number quoted by Doljack and Hoffman ${ }^{7}$ for nickel, $\Delta_{\mathrm{Ni}}$ $=0.08 \mathrm{~nm}(25 \%$ of the interatomic distance $)$. If neighboring grains would not interact, the average extra distance between two atoms on each side of the grain boundary would be $50 \%$ of the interatomic distance. Hence any value for $\Delta$ less than $50 \%$ of the interatomic distance is physically feasible.

For stoichiometric films we expected a priori a stress development with thickness comparable with the observed behavior in chromium films. Surprisingly, also understoichiometric films, which contain a mixture of the hexagonal $\mathrm{Cr}_{2} \mathrm{~N}$ phase and the cubic $\mathrm{CrN}$ phase, show a qualitatively equal development of stress with thickness.

\section{CONCLUSIONS}

In conclusion stress in $\mathrm{CrN}$ films is described by the sum of three contributions. Tensile stress is generated at grain boundaries and during cooling from the elevated deposition temperature to RT. Compressive stress is generated by ion peening. The tensile stress due to grain boundaries depends on the height in the film. At each height in the film it is proportional to the number of grains per unit length at that height, leading to a stress gradient over the thickness of the film. The evolution of grain size and stress with thickness is described by the same power law. From this common description an amount of grain boundary shrinking of $0.08 \mathrm{~nm}$ is deduced. The thermal tensile stress is not dependent on film thickness. Compressive stress is caused by ion peening. Also, these latter two effects have been isolated.

\section{ACKNOWLEDGMENTS}

This research was carried out under project No. MC 7.02124 within the framework of the Strategic Research Pro- gram of the Netherlands Institute for Metals Research. X-ray diffraction was performed by N. M. van der Pers and EPMA by J. Kiersch, both of the Department of Materials Science and Engineering.

${ }^{1}$ B. A. Movchan and A. V. Demchishin, Fiz. Met. Metalloved. 28, 83 (1969).

${ }^{2}$ I. Petrov, P. B. Barna, L. Hultman, and J. E. Greene, J. Vac. Sci. Technol. A 21, S117 (2003).

${ }^{3}$ J. P. Lokker, Ph.D. thesis, TU Delft, 2002.

${ }^{4}$ F. Spaepen, Acta Mater. 48, 31 (2000).

${ }^{5}$ S. C. Seel, C. V. Thompson, S. J. Hearne, and J. A. Floro, J. Appl. Phys. 88, 7079 (2000)

${ }^{6}$ J. A. Floro, S. J. Hearne, J. A. Hunter, P. Kotula, E. Chason, S. C. Seel, and C. V. Thompson, J. Appl. Phys. 89, 4886 (2001).

${ }^{7}$ F. A. Doljack and R. W. Hoffman, Thin Solid Films 12, 71 (1972).

${ }^{8}$ A. J. Dammers and S. Radelaar, Mater. Sci. Forum 94-96, 772 (1992).

${ }^{9}$ Paritosh, D. J. Srolovitz, C. C. Bataile, X. Li, and J. Butler, Acta Mater. 47, 2269 (1999).

${ }^{10}$ G. C. A. M. Janssen, A. J. Dammers, V. G. M. Sivel, and W. R. Wang, Appl. Phys. Lett. 83, 3287 (2003)

${ }^{11}$ F. M. D'Heurle, Metall. Trans. 1, 725 (1970).

${ }^{12}$ I. Petrov, L. Hultman, U. Helmersson, J.-E. Sundgren, and J. E. Greene, Thin Solid Films 169, 299 (1989).

${ }^{13}$ G. C. A. M. Janssen and J.-D. Kamminga, Appl. Phys. Lett. 85, 3086 (2004).

${ }^{14}$ R. Hoy, W. G. Sloof, and G. C. A. M. Janssen, Surf. Coat. Technol. 179, 215 (2004)

${ }^{15}$ P. A. Flinn, D. S. Gardner, and W. D. Nix, IEEE Trans. Electron Devices ED-34, 689 (1987)

${ }^{16}$ M. F. Doerner and W. D. Nix, CRC Crit. Rev. Solid State Mater. Sci. 14, 225 (1988).

${ }^{17}$ C. A. Volkert, C. F. Alofs, and J. R. Liefting, J. Mater. Res. 9, 1147 (1994).

${ }^{18}$ Handbook of Tribology, edited by B. Bhushan and B. K. Gupta (McGrawHill, New York, 1991).

${ }^{19}$ Plenum Press Handbooks of High Temperature Materials, No. 2 Properties Index, edited by G. V. Samsonov (Plenum, New York, 1964).

${ }^{20}$ D. Gall, C.-G. Shin, T. Spila, M. Odén, M. J. H. Senna, J. E. Greene, and I. Petrov, J. Appl. Phys. 91, 3589 (2002). 